\title{
Primary Undifferentiated Pleomorphic Sarcoma of the Colon Mesentery
}

\author{
Joo Hyun Lee, Dong Baek Kang, Won Cheol Park \\ Department of Surgery, Wonkwang University Hospital, Wonkwang University School of Medicine, Iksan, Korea
}

An undifferentiated pleomorphic sarcoma (UPS), also known as a malignant fibrous histiocytoma in the past, commonly involves the soft tissue of the extremities and the retroperitoneum. However, a primary UPS of the colon mesentery is very rare. A 69-year-old male patient visited our outpatient department for treatment of an enlarged, palpable mass in the right lower quadrant (RLQ). Computed tomography showed a $15-\mathrm{cm}$ multilobulated, heterogeneous, enhanced mass in the RLQ, which we suspected originated from the colon. He underwent a right hemicolectomy, and the pathologic result was a colon mesenteric UPS. We report a rare case of a primary UPS of the colon mesentery and discuss the characteristics of this neoplasm in reference to the literature.

Keywords: Undifferentiated pleomorphic sarcoma; Malignant fibrous histiocytoma; Mesentery

\section{INTRODUCTION}

An undifferentiated pleomorphic sarcoma (UPS), also known as a malignant fibrous histiocytoma (MFH) in the past, commonly involves the soft tissue of the extremities and the retroperitoneum [1]. However, a primary UPS of the colon mesentery is very rare. We report a rare case of a primary UPS of the colon mesentery and discuss the characteristics of this neoplasm in reference to the literature.

\section{CASE REPORT}

A 69-year-old male patient visited our outpatient department for treatment of an enlarged, palpable mass in the right lower quadrant (RLQ) area, which had been present for several months. He complained of mild, dull, and constant abdominal pain, but had no fever, diarrhea, or constipation. A physical examination re-

Received: January 4, 2018 - Accepted: March 11, 2018

Correspondence to: Won Cheol Park, M.D.

Department of Surgery, Wonkwang University Hospital, Wonkwang University School of Medicine, 460 Iksan-daero, Iksan 54538, Korea

Tel: +82-63-859-1496, Fax: +82-63-855-2386

E-mail: parkwc@wku.ac.kr

ORCID code: https://orcid.org/0000-0002-2389-5738

(C) 2019 The Korean Society of Coloproctology

This is an open-access article distributed under the terms of the Creative Commons Attribution NonCommercial License (http://creativecommons.org/licenses/by-nc/4.0) which permits unrestricted noncommercial use, distribution, and reproduction in any medium, provided the original work is properly cited. vealed a huge, firm, palpable mass in the RLQ of the abdomen. The patient's blood pressure was $107 / 78 \mathrm{mmHg}$, his pulse rate was 78 beats $/ \mathrm{min}$, and his body temperature was $36.7^{\circ} \mathrm{C}$. In the complete blood count, hemoglobin was $11.2 \mathrm{~g} / \mathrm{dL}$, the white blood cell count was $22.8 \times 10^{3} / \mathrm{mm}^{3}$. The C-reactive protein was $110.28 \mathrm{mg} / \mathrm{L}$, and the carcinoembryonic antigen was $1.53 \mathrm{ng} / \mathrm{mL}$; the other blood chemistry parameters were within normal limits.

He underwent computed tomography (CT), which revealed a 15-cm multilobulated, heterogeneous, enhanced mass in the RLQ, which we suspected originated from the colon (Fig. 1). The patient underwent a laparotomy in September 2017. Operative findings revealed a huge solid mass surrounding the ascending colon. The mass occupied the mesentery from the cecum to the hepatic flexure. We next performed a right hemicolectomy (Fig. 2). The pathology results revealed an UPS of the ascending colon mesentery that had invaded the visceral peritoneum of the colon. Immunohistochemical staining for alpha 1-antichymotrypsin and vimentin were positive, and those for CD34, DOG-1, c-Kit, S-100 protein, smooth muscle actin (SMA), desmin, CD31, HMB45, calretinin, LCA, and PanCK were negative (Fig. 3). The proximal resection margin was $6 \mathrm{~cm}$, the distal margin was $8 \mathrm{~cm}$, and all resection margins were clear. The final diagnosis was UPS originating from the colon mesentery. However, no evidence of distance metastases was found, and the surgical resection margin was clear. We recommended chemotherapy and radiotherapy because the tumor was huge and had invaded the visceral peritoneum, but the patient refused. The patient was discharged on postoperative day 
Volume 35, Number 3, 2019

Ann Coloproctol 2019;35(3):152-154 Coloproctology

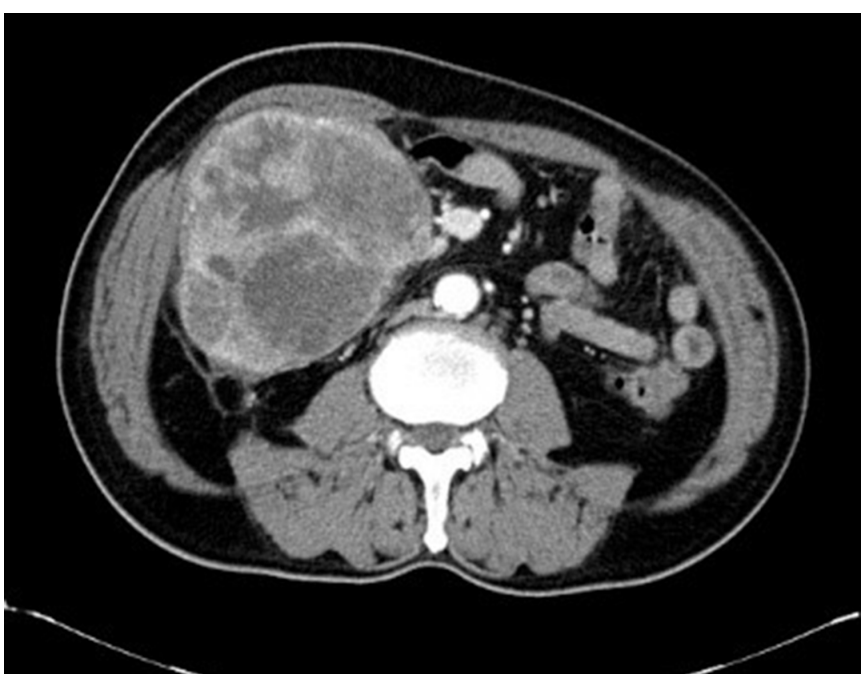

Fig. 1. The computed tomography findings of undifferentiated pleomorphic sarcoma show a $15-\mathrm{cm}$ multilobulated heterogeneous enhanced mass in the right lower quadrant. We suspect that the mass originated from the colon.

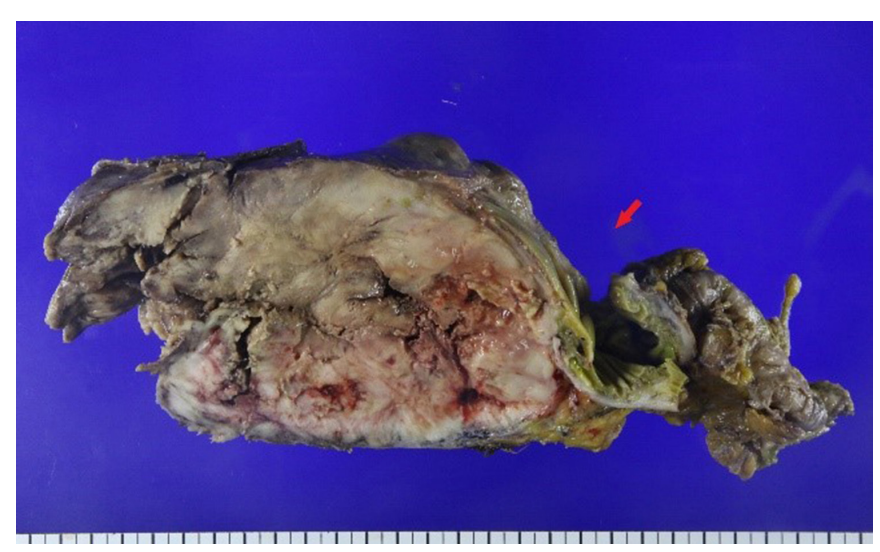

Fig. 2. Gross pathologic findings of undifferentiated pleomorphic sarcoma show a huge mass measuring about $18 \mathrm{~cm} \times 14 \mathrm{~cm} \times 13.5$ $\mathrm{cm}$. The mass originated from the colon mesentery and encased the ascending colon. The red arrow indicates the cecal lumen.

11 without any postoperative complications. Unfortunately, he revisited our emergency department for abdominal pain after 2 months, and CT revealed multiple metastases of the tumor. The patient subsequently refused all treatment.

The Institutional Review Board of Wonkwang University Hospital approved this study and waived the informed consent requirement (WKUH 2018-09-030).

\section{DISCUSSION}

UPS, also known as MFH in the past, is defined as a high-grade pleomorphic neoplasm that does not show a definable line of dif-
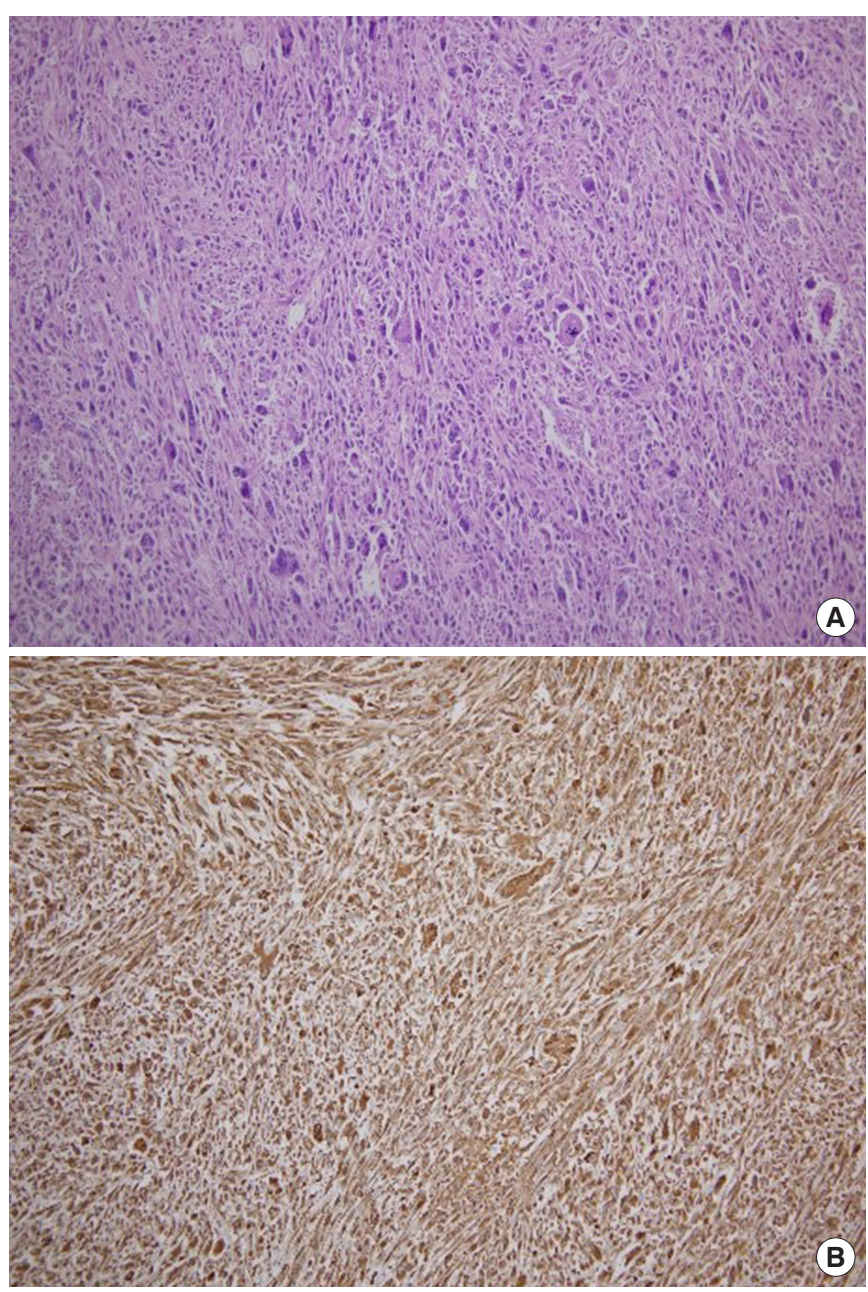

Fig. 3. (A) Microscopic pathologic findings show a storiform arrangement consisting of spindle cells and histiocyte-like cells (H\&E, $\times 100$ ). (B) The immunohistochemical staining was positive for alpha1-antichymotrypsin $(\times 100)$.

ferentiation under current techniques [1]. MFH was first described in 1963, as a malignant histiocytoma and a fibrous xanthoma [2]. In 1978, MFH was divided into four histological subtypes: myxoid, storiform-pleomorphic, giant-cell, and inflammatory [3]. According to the 2002 World Health Organization classification, these 4 subtypes of MFH were reclassified. The term "storiform-pleomorphic MFH" was replaced with "UPS," "giantcell" with "UPS with giant-cells," and "inflammatory" with "UPS with prominent inflammation." Furthermore, these 3 types were considered fibrous histiocytic tumors. "Myxoid MFH" was renamed to "myxofibrosarcoma" and classified into the myofibroblastic category [1].

UPS has a peak incidence in the sixth and the seventh decades of life and usually involves the extremities (lower extremities, 49\%; upper extremities, 19\%), retroperitoneum, and abdominal cavity (16\%) [3]. UPS at other sites is very rare. When the tumor 
involves the mesentery, patients often complain of abdominal pain, dyspepsia, abdominal distension, fatigue, weakness, weight loss, and a palpable mass. However, symptoms are nonspecific and occur after disease progression, making preoperative diagnosis difficult $[3,4]$. The CT findings of UPS usually show a wellcircumscribed and homogenous mass or a mass with internal low density, owing to necrosis or hemorrhage $[5,6]$. In our case, CT revealed a heterogeneous enhanced mass, owing to necrosis. Pathologically, UPS is characterized by a haphazard and storiform arrangement of highly pleomorphic, spindle-shaped cells with bizarre cytology and pleomorphic nuclear atypia. Immunohistochemical staining for vimentin, actin, CD68, alpha 1-antitrypsin, alpha 1-antichymotrypsin and laminin mRNA are frequently positive [1,7-9].

Diagnosing UPS requires the exclusion of other tumors that have specific lines of differentiation, and the differential diagnosis includes a gastrointestinal stromal tumor, pleomorphic liposarcoma (LPS), pleomorphic leiomyosarcoma (LMS), pleomorphic rhabdomyosarcoma (RMS), dedifferentiated LPS, myxofibrosarcoma, poorly differentiated carcinoma, and melanoma. Some special stainings assist the differential diagnosis of these tumors. Making a diagnosis of pleomorphic LPS depends on the identification of multivacuolated lipoblasts, and LPS is frequently positive for SMA, S-100 protein, keratins, and desmin. Pleomorphic LMS and RMS are myogenic differentiated tumors that are both positive for desmin. Diagnosing the former depends on positivity for $\mathrm{h}$-caldesmon while diagnosing the latter depends on the presence of a skeletal muscle marker, such as myf- 4 or fast myosin. Positivity for the S-100 protein and other melanocytic markers is useful in diagnosing a melanoma $[1,7,8]$. In our case, the tumor was positive for alpha 1-antichymotrypsin and vimentin.

The standard treatment of UPS is an early and complete surgical resection with negative resection margins. The role of adjuvant radiotherapy is well established in the treatment of patients with UPS of the extremities, but the effects of radiotherapy for treating patients with a mesenteric UPS remain unclear. Chemotherapy using doxorubicin and ifosfamide is commonly reserved for patients with unresectable or metastatic tumors $[7,10]$. The prognosis of MFH is poor, and the 2-year survival rate is $60 \%$, with a recurrence rate of $44 \%$ and a metastatic rate of $42 \%$ [3]. The prognosis for patients with mesenteric UPS is unclear, although most reports suggested poor prognoses.

In conclusion, we report a case of primary UPS of the colon mes- entery that was treated by using a right hemicolectomy. Primary UPS of the colon mesentery is a very rare disease, but the small possibility of this disease must be considered while making a diagnosis.

\section{CONFLICT OF INTEREST}

No potential conflict of interest relevant to this article was reported.

\section{REFERENCES}

1. Fletcher CD, Unni KK, Mertens F. editors. World Health Organization classification of tumours. Pathology and genetics of tumours of soft tissue and bone. Lyon: IARC Press; 2002.

2. Ozzello L, Stout AP, Murray MR. Cultural characteristics of malignant histiocytomas and fibrous xanthomas. Cancer 1963;16: 331-44.

3. Weiss SW, Enzinger FM. Malignant fibrous histiocytoma: an analysis of 200 cases. Cancer 1978;41:2250-66.

4. Park SB, Hong SK. A case of recurrent malignant fibrous histiocytoma of the mesentery. Int J Surg Case Rep 2016;25:106-9.

5. Goldman SM, Hartman DS, Weiss SW. The varied radiographic manifestations of retroperitoneal malignant fibrous histiocytoma revealed through 27 cases. J Urol 1986;135:33-8.

6. Park JH, Yeon JW, Han EM, Jang SK, Kang SM, Ahn IO. Primary malignant fibrous histiocytoma of the mesentery: a case report. J Korean Radiol Soc 2007;57:549-52.

7. Nascimento AF, Raut CP. Diagnosis and management of pleomorphic sarcomas (so-called "MFH") in adults. J Surg Oncol 2008;97: 330-9.

8. Diaz-Beveridge R, Melian M, Zac C, Navarro E, Akhoundova D, Chrivella M, et al. Primary mesenteric undifferentiated pleomorphic sarcoma masquerading as a colon carcinoma: a case report and review of the literature. Case Rep Oncol Med 2015;2015: 532656.

9. Soini Y, Autio-Harmainen H. Tumor cells of malignant fibrous histiocytomas express mRNA for laminin. Am J Pathol 1991;139: 1061-8.

10. Sleijfer S, Seynaeve C, Verweij J. Using single-agent therapy in adult patients with advanced soft tissue sarcoma can still be considered standard care. Oncologist 2005;10:833-41. 